\title{
Open Data and the Danger of Sympathetic Magic
}

Brian Ballsun-Stanton and Georgia Burnett

\begin{abstract}
This chapter provides a minimal normative framework for discussing the desired attributes of a published dataset using Lakatos' philosophy of science and the metaphor of sympathetic magic. When researchers publish their results, journals and granting agencies increasingly want datasets opened alongside. These datasets may vary in quality, reusability, and comprehensiveness. Yet, without a clear knowledge of how the research methodology and analysis affects the production of the raw data, any future attempts to reproduce analysis will produce the same magical results only by following the same steps. While the requirement for Open Data in government funded research can provide an excellent basis for future research, not all Open Data is created equal. Releasing the methods of analysis alongside a dataset of high quality will also allow for lower technical difficulties when reusing or remixing the data. Then data, collected once, may be reused in multiple projects for a significant research impact. And to some, we can show that data is, indeed, magic.
\end{abstract}

Key words: Open Data; Archaeology; Data Standards and Quality; Reproducibility.

\section{Introduction}

Effective data management is an important part of ensuring open access to publicly funded research data. Data management planning from the beginning of a research project helps to

Brian Ballsun-Stanton

Macquarie University, Faculty of Arts 16 University Ave, Sydney NSW 2109, e-mail: brian. ballsun-Stanton@mq.edu.au

Georgia Burnett

Macquarie University, Department of Ancient History, Faculty of Arts, 16 University Ave, Sydney NSW 2109, e-mail: georiga. burnett@hdr.mq.edu.au 
outline how data will be collected, formatted, described, stored and shared throughout, and beyond, the project lifecycle, (Australian Research Council. 2016).

Archaeologists generally gather unique data which can seldom be gathered again. A critical issue for archaeological data is its ad hoc nature: where methods are applied according to transitory and unanticipated needs and recorded incrementally over field seasons by teams and individuals that pass in and out of the project. While other archaeologists will be aware of this issue in their professional experience, an explicit acknowledgement and recording of its impact on data may help to encourage an intentional and consistent approach to data quality. Here, we present some guidelines concerning data quality, which will help datasets to more easily reused by source communities and other researchers. By embracing this approach, data can benefit multiple groups, including community members, other researchers, and the originating researcher's own future projects.

This chapter first discusses the use of Open Data within archaeological disciplines. James defines Open Data as "data that can be freely used, shared and built-on by anyone, anywhere, for any purpose," 2013. We then connect Open Data and its use to the metaphor of sympathetic magic. Sympathetic magic refers to the act of manipulating the image of something to manipulate the thing itself. Here, the metaphor of sympathetic magic is useful in describing current Open Data publication objectives: for many researchers and granting agencies, the fact of the data release is more important than the utility of the release. This emphasis on image - that is, the presentation of the data as a object-rather than substance is a good fit for the metaphor of sympathetic magic. Lakatos, a philosopher of science, in his lecture titled "Science and Pseudoscience", differentiated between progressive and degenerate 1 research programs (1978). The progressive research program distinguishes itself by the creation of novel and unanticipated facts; where degenerate research programs are only able to shuffle and regurgitate that which is already known. This philosophy also maps well onto a discussion of the quality of datasets.

Falsifiable predictions need not only data, but knowledge of the data structures and collection methodologies used. Open Data must have: 1) the data itself; 2) consistent and extensible data structures; 3 ) and documented collection and analysis methods for the data. The data must be shareable, remixable, and reproducible (Nature, 2016). Those who reuse the data must be able to achieve the same conclusions without instruction or input from the original researchers. Open Data, when it is remixed or reused, must be able to generate novel and surprising facts, thereby echoing Lakatos' definition of a progressive research program. Data must exist outside the instructions of the creator. Useful datasets, therefore, must provide working tests and documentation to survive the original author's death or retirement.

\footnotetext{
${ }^{1}$ In this chapter we will use the term problematic, when referring to datasets to avoid unwanted connotations.
} 
Academia has long adopted "standing on the shoulders of giants" as one of its mottoes. We should not need to trust the arguments of our predecessors in this gradual building of knowledge. We should be able to double check their conclusions through our own analysis of their data. We should be able to add to the data they produced, secure in the knowledge that the dataset only grows more reliable and robust with new additions. We agree with the argument from a Nature editorial that proclaims: "Researchers need to be obliged to document and manage their data with as much professionalism as they devote to their experiments. And they should receive greater support in this endeavour than they are afforded at present," (Nature, 2008).

Peer review, from a journal's perspective, must, "[serve] as a filter in distinguishing between 'good' and 'bad' science," (Horbach and Halffman, 2018). However, the specific mechanisms of this demarcation are under debate. One mechanism, which we wish to highlight here, is that of results free peer review (see Findley et al, 2016). Journals using this methodology add a step which is, "[A] review of manuscript, excluding any results or discussion of results," (BMC Psychology, 2017). Our proposed framework for usable or progressive Open Data is a way of assessing the metadata without needing to inspect the data and could provide a useful heuristic for this style of peer review. Specifically, reviewers could inspect the intentional, articulate, creation of test cases and well-documented data structures as metadata without needing to examine the specific conclusions or data of the publication.

We conclude with a thorough examination of progressive and problematic Open Data, creating a normative framework for what makes usable Open Data, the value of documentation and test driven data collection, and some traps of implicit knowledge transfer. Usable Open Data must:

1. be easily transformed into other formats;

2. be easily sorted and filtered;

3. be easily referenced into extant lookup domains (like places, people);

4. be easily aggregated into summary statistics.

Our recommendation of test driven development for computational academic research reduces error rates in analysis and provides a critical check of the quality of data structures before destructive data collection occurs. An awareness of tacit knowledge transfer through the movement of graduate students between labs (Galison, 1997) and the lived experiences of researchers provides another check on the necessity and quality of documentation, and offers the final constraint on the ultimate use of the collected resource. 


\section{Open Data and Archaeology}

Open Data requires that published data must conform to the following rules:

1. The work must be in the public domain or provided under an open license;

2. The work must be provided as a whole and at no more than a reasonable one-time reproduction cost, and should be downloadable via the Internet without charge;

3. The work must be provided in a form readily processable by a computer and where the individual elements of the work can be easily accessed and modified;

4. The work must be provided in an [non-proprietary] open format (Open Knowledge International, 2016).

The goals of Open Data are: discoverability, preservation, and access to research data (Beck and Neylon, 2012). Archaeological data should be discoverable through the web in order to promote reuse. Data should be preserved in appropriate repositories, and be configured in accessible formats with instructive metadata to ensure longterm access by any stakeholders, allowing for large scale studies outside the scope of single projects. It is important to note the scope of this chapter: we are not arguing for open access of publication, though we strongly support open access to research outputs in general as a necessary precondition for Open Data Instead, we want to focus on Open Data and the requirements for useful and effective sharing of scholastic and archaeological data.

Within current professional norms ${ }^{3}$, archaeological data is "shared with other scholars but infrequently, informally, when time permits, and sometimes only when someone asks," (Peebles, 2010). It is rare to publish complete, online datasets alongside traditional publications in archaeology, and rarer still to ensure reproducible data analysis as part of standard review processes (Costa et al, 2013). Print press, the traditional mode of publication for archaeological research, discourages publishing full datasets as a factor of cost. While routes are available for researchers who wish to publish their full datasets as citable publications (see datasets in Open Context and data papers in the Journal of Open Archaeology Data, among others) these are effectively their own publications, without much formal link from the original publication to the data. There exists a tension between journals such as Nature which requires:

${ }^{2}$ See https://libguides.mq.edu.au/Open_Access Archive: https://perma.cc/XG9W-YLPN for an Australian unfunded open access approach

${ }^{3}$ For a humorous illustration of current data sharing and management norms, watch "Data Sharing and Management Snafu in 3 Short Acts" by the NYU Health Sciences Library https:// youtu.be/N2zK3sAtr-4 Archive: https://web.archive.org/web/20170718041644/https: $/ /$ www youtube . com/watch?v=N2zK3sAtr -4 
.. [T] hat others should be able to replicate and build upon the authors' published claims. A condition of publication in a Nature Research journal is that authors are required to make materials, data, source code, and associated protocols promptly available to readers without undue qualifications (Nature Publishing Group 2018).

This policy stands in contrast to the Society for American Archaeology's policy which notes that while,

Authors are strongly encouraged to make their data available through ... sites that support both data preservation and allow access as appropriate. ... Supplemental material is not an acceptable place to provide raw data related to publication, as this is not archived with the body of the journal article (Society for American Archaeology 2018).

Note the distinction between Nature's policy of maximum data availability, and the SAA Editorial policy of encouraging data availability at two stages of remove: The journal, the online (but non-archived) supplemental material, and then finally the independent site which can hold the raw data. In this space, archaeology is lagging behind the premier scientific publications, and still is focused around the idea of selected samples. That selected sample is data which most effectively demonstrates the conclusions drawn by the author. Current professional reward structures and norms within archaeology also discourage deviation from mainstream publication avenues, such as journals and monographs. Additionally, many researchers do not have the time, money or technical know-how to efficiently and effectively share their raw data (Frank et al, 2015). By the time attention turns to the long-term preservation of research data, researchers lack the time to spend on preparing the data for publication, or the funds to offload this task to assistants or professional staff. The difference between required usable and peer reviewed datasets with the article and encouraged publication in institutional archives is the explicit rejection that access to the data is needed to support archaeological claims. This distinction is exactly the issue we raise in this chapter. The data, deposited as an afterthought in an instutional repository, has no required checks to see if it is useful and does not function in a persuasive relationship with published research to support the conclusions of the authors or to form a solid and peer-reviewed foundation for the research of future researchers.

Open Data allows for archaeologists to engage in more rigorous research. By opening full datasets to outside scrutiny, all archaeologists (including the original investigator) will benefit. The provision of well-documented datasets provides the data itself, as well as practical learning opportunities by allowing the spread of methodologies and analyses. These methods, not needing reverse engineering from word-limited papers, can also serve to improve the quality of other researchers' publications. Traditional paper-based publishing tends to be limited by cost, and discussion and interpretation of findings is prioritized. With mixed media publishing and publishing complete datasets as fundamental components of a publication, rather than demonstrative samples that support the conclusions made by the primary investigator, researchers can 
allow for a more rigorous review process of their research pipeline: data, analytical methods, computational implementation of analysis, and conclusions. By providing complete datasets with a firm understanding of Open Data principles, archaeologists can more thoroughly check, integrate, and extend the conclusions of their peers, thereby offering more research opportunities and citation opportunities for the same initial investment of resources.

Sharing archaeological data is neither new nor pioneering (Flannery, 1982). There is a strong ethical case for data sharing to become a professional norm within archaeology, at least where sharing is appropriate (with exceptions noted below). Archaeological excavation is a destructive methodology that cannot be repeated, as excavation entails the destruction of any archaeological contexts. Survey is largely non-repeatable as archaeologists cannot foresee natural changes in the landscape. Both forms usually result in the archaeologists' dataset as the only surviving record of the site. In the case of academic archaeology, which is largely taxpayer funded through government grants and awards, withholding datasets and their associated research represents a significant waste of public spending. Releasing these datasets for remixing or reuse by other researchers ensure more value for money, as the data can be integrated into projects outside the original project's scope. Outside of academic research, many archaeological projects are performed within the parameters of commercial or salvage archaeology, usually under the goal of public good outcomes (North 2006). It is therefore imperative that all of these datasets, both academic and commercial, remain discoverable, available, and accessible to future researchers. The issue of accessibility is very severe, as "the odds of a data set being extant [fall] by 17\% per year," (Vines et al, 2014). When left without proper management, unique and irreplaceable contextual data can remain inaccessible on personal drives, lost to bit rot, isolated on a private server, or be locked behind costly paywalls. Furthermore, Kansa argues that researchers dealing in archaeology "should not continue to tolerate the personal, self-aggrandizing appropriation of cultural heritage that comes with data hoarding," 2012 Archaeology needs to build transparent data workflows (quantiative or qualitative data notwithstanding) to publish and treat the data as a fundamental aspect of a research work, instead of the current practice of, "bolt-on data reproduciblity," (Marwick, 2017). Much of the expense of publishing data exists due to this bolt-on mindset. Collecting data with these goals in mind can improve data quality without much impacting the total difficulty of collecting and using the data.

With culturally sensitive materials, however, there are ethical issues with the current permissive definition of Open Data. While popular conceptions of archaeology define it as the study of past cultures, many archaeologists work with communities whose culture and relative access protocols remain present. Many indigenous cultures have active and restrictive cultural access protocols and knowledge systems surrounding their own cultural materials and landscapes which fall outside the definition of open that is generally used. While adopting the strong definition of Open Data might be ethical in some cases, imposing this definition as a universal code in archaeology 
is unacceptable (Kansa, 2012). This conflict represents a difficult challenge for archaeology, but not an insurmountable one. For example, data services like Murkutu 4 control user access to datasets as required by the indigenous cultural protocols, while ensuring the spirit of Open Data: discoverability, preservation, and access. With Murkutu, data owners can control access to specific materials as defined by their own knowledge systems and access requirements. Yet, while cultural access controls restrict user-view, the data as a whole remains discoverable, preserved as a whole dataset, and the archival system curates and updates its formats in perpetuity, ensuring long-term access. As another implementation of ethical data access restriction, Data Archiving and Networking Services (DANS), a Netherlands-based service, allows for offline data preservation, but maintains a point of access for the dataset. They provide data archiving as a service to ensure the data is preserved, discoverable, and accessible within the limits of its use.

While these instances of embargoed data restrict universal access to the data, the data is still rendered according to Open Data principles and guidelines. These principles ensure that this data will remain in usable formats with adequate documentation so that inheritors can expand and integrate the data into any future community project or initiative. Rather than dismissing Open Data for it's permissive and Western handling of the idea of openness (Kersel 2016), exploring, understanding and navigating these ethical concerns should be at the forefront of Open Data research for archaeology.

Outside the more specialised repositories mentioned above, there are many avenues for archaeologists to make their data available, or at least discoverable, online for others' use. Archives like the Archaeology Data Service (ADS) ${ }^{5}$ in the United Kingdom, DANS in the Netherlands, and the Digital Archaeological Record (tDAR) 6 in the United States provide services that facilitate long-term, sustainable data preservation. By its requirement of non-proprietary formats, the Open Data movement has been instrumental in encouraging archives to provide data curation services. Typically, those wishing to deposit their data into these repositories are encouraged by the guidelines to use standard file formats.

Like many data repositories, tDAR, the ADS, and DANS have guidelines which promote Open Data. For example, the ADS website lists recommended and accepted file formats which the archive supports for a variety of file types, preferring files that are for the foreseeable future, industry standard; as does DANS, with the added incentive of guaranteeing long-term preservation of files 7 By providing long-term support for file formats, these repositories ensure their datasets will not be trapped

4 http://mukurtu.org/Archive: https://perma.cc/KE4E-HYPE

5 http://archaeologydataservice.ac.uk/ Archive: https://perma.cc/A74N-B4P8

6 https://core.tdar.org/Archive: https://perma.cc/ZWM4-EJNA

7 For more on depositing data to the ADS see http://archaeologydataservice.ac.uk/ advice/DepositingData Archive: https://perma.cc/3MAT-595Z For more on depositing data to DANS see https://dans.knaw.nl/en/deposit/information-about-depositing-data Archive: https://perma.cc/4VR7-NWE3 
in obsolete formats for the foreseeable future. Most repositories also promote Open Data in their documentation standards. These requirements range from permissive to strictly regulated, though all provide essential recommendations to how data can be made more accessible to others by documenting the semantic structure of the dataset.

There are, however, systemic challenges to the adoption and adaptation of technological change in archaeology. Increasingly, grant applications require data management plans. These requirements are sometimes vague and permissive as to their expected outcomes. Granting agencies are imposing these standards as unfunded mandates: there are seldom additional funds to accommodate the costs of long-term digital archival storage (Lynch, 2008; Nature, 2008).

Archaeological datasets are also typically developed ad hoc according to transitory and unanticipated needs, recorded incrementally over field seasons by teams and individuals that pass in and out of the project. This combination of factors often results in a lack of data modelling, metadata documentation, or update strategies for these resulting databases (Carver and Lang, 2012, Kansa and Kansa, 2013). The resulting datasets require extensive clean up, editing, and review once a project is finished. This clean-up, too, often relies on implicit knowledge gained from personal engagement with a project, rather than documented guidelines or structures. Typically, quality data management requires a level of technical skill that is not routinely taught in archaeology degrees. Data management skills are often an optional undertaking made at the discretion of an individual, and require a substantial investment of time and money.

Some of these problems outlined above were encountered by ArchaeoInf, a Germanbased project that ran between 2009 and 2013. ArchaeoInf aimed at providing a: "single point of access for primary data acquired through excavations and field surveys," (ArcheoInf-Redaktion, 2010). Their experience developing the repository gave an interesting insight into the mindset of archaeologists transitioning to digital modes of preservation. Carver identified a fundamental obstacle in developing their repository: an unwillingness to submit data to the repository, even for testing purposes. They identified this reluctance as Technofrust, a German word embodying people's frustration with technology, largely to do with "unrealistic expectations of what computers can do," 2012 While experience had shown that once the initial investment into a specific digital skill is made, it becomes indispensable, training is often considered a significant cost of both time and money. There is, then, a decision made on a reasonable trade off between ease-of-use and a more technically complex system that might be better suited to the job. Without a background education in Information Technology principles, the immediate or long-term benefits of data modelling and database design are not obvious to many archaeologists.

The research advantages of Open Data are best understood through the successful projects it has produced. One of the cornerstone projects, whose success was directly attributed to Open Data, was a major collation of archaeozoological data 
from 200,000 faunal specimens spanning several distinct projects from seventeen neolithic sites in Turkey (Arbuckle et al, 2014). In consultation with the data publication service Open Context, the project's output provides an exceptional example of the benefits of web-based data publication of primary datasets for exploring major questions in archaeology. This project provided revisions and answers to questions regarding Neolithic animal economies that would be outside the scope of any individual research project. Additionally, the outcomes demonstrate the need not only for raw datasets to be made available, but for that data to be accessible for other archaeologists to use and extend upon. Undocumented data that is made available online, while better than no data, has a number of barriers to entry by outsiders. Facts, as we will discuss, cannot exist in a vacuum; an understanding of how data is produced, encoded, and documented is essential to understanding it.

\section{What is Sympathetic Magic?}

PERHAPS the most familiar application of the principle that like produces like is the attempt which has been made by many peoples in many ages to injure or destroy an enemy by injuring or destroying an image of him, in the belief that, just as the image suffers, so does the man, and that when it perishes he must die (Frazer. 1922).

Sympathetic Magic refers to the principle of "like produces like" (Frazer, 1922): that likeness between originals and replicas make them functionally the same. For the purpose of this paper, we use the idea of like-produces-like as a metaphor of problematic Open Data. While the practice of producing Open Data is commendable, there are often no checks on the data's reusability. Merely presenting the data with the hopes that someone will find it useful is a sympathetic remedy that does not help others fundamentally understand the dataset. The true value in Open Data is its extensibility and interoperability with other like datasets. However, without a clear knowledge of how methodology, theory, and analysis affects the production of raw data, any future attempts to reproduce conclusions must follow in the exact same path to produce the results: like produces like. We propose that such a conflation between conclusions and research data has occurred in the usual practice of publishing and requiring Open Data.

The reproducibility problem can also be called a consequence of sympathetic magic. The recent reproducibility project in psychology is finding the practice of reproduction quite difficult to achieve:

Reproducibility is a defining feature of science, but the extent to which it characterizes current research is unknown. ... Replication effects ... were half the magnitude of original effects ..., representing a substantial decline (Open Source Collaboration 2015).

Unfortunately, merely presenting consumers of the research with the data from the research will do very little in helping them to understand and extend the data. We 
supply the image of the already analyzed data, expecting it to be useful to produce novel facts. We can see steps towards the sharing of methods along with data with protocols.io (Edmunds, 2016) and the Open Science Framework, among others. To use protocols.io as an example, it functions as an electronic lab notebook, itemising each step in a computationally readable form, documenting results along with steps taken, and creating a timestamped and attestable framework to present those methods and data within. These documents can then be included in methods sections of published papers to allow academics to explore the exact steps taken so that they may better understand or reproduce the data (protocols.io, 2018). Archaeologists can perform similar analyses, using R notebooks (Sobotkova, 2018) paired with dedicated and open data collection methods (Sobotkova and Janouchova, 2018) can create research which can be deeply inspected, shared, and repurposed by other archaeologists for their own research purposes. Sobotkova's workflow presents the data collection method in a well described format: the data collection tool has an XML file which builds the data collection; has domains for each column by virtue of sharing the source code ${ }^{8}$ and software which builds the data collection; and a containerised workflow which takes the data as published and performs a public analysis on it. We are not simply presented the clean data, but all of the artifacts which created the data and the conclusions drawn from said data.

Open Data must stand on a tripod:

1. The data itself;

2. Consistent and extensible data structures; and

3. Documented collection and analysis methods for the data.

Without the structures and methods, the shared data is merely an image; an image which cannot be altered or extended.

\subsection{Sympathetic Magic and Open Data}

The presentation of good and useful Open Data is not only a challenge for archaeologists. Looking at governmental data repositories, we can see similar issues. Open Data published through the various government data portals varies in quality and professionalism (Yang and Kankanhalli, 2013, p. 648). Well-articulated datasets include data, metadata which at the very least notes what each column should contain and if it is a constrained vocabulary, and examples of use. A good example of this presentation is a Jupyter or R Notebook, which includes as part of its files a readme, explicit license (CC-BY or better), the raw data in CSV files, discussion and links

${ }^{8}$ Source code, or "code" is used to build software, but can also be used as a sort of "script" or recipe to run an analysis (Marwick, 2017). 
to the data collection methodology, and analyses of the data. In this regard, the data analysis is important not only for the research it performs, but for how it demonstrates and encodes the intended uses of the data (see Sobotkova 2018).

Common flaws of datasets include ones that elide the how and why of their collection. Specifically, how was the data collected (the data collection methodology) or why they chose to include this specific set of data. While the readme files in the collections may note a methodology, it is seldom to the level of specificity to allow someone else to collect data in the same manner for the same purpose.

Data, as published, is usually missing a frame contextualizing it within a community of datasets. That is, when the data is deposited, little thought is given to the extensibility of the data; rather, the value is placed on the support the data lends to the conclusions of the primary investigator. We challenge that the fundamental test of a dataset should be the question: "can we add to this dataset?" The answer becomes the differentiating factor between a single use tool for the original researcher or an extensible resource for the whole community to profit from. If the dataset is of sufficient quality to allow appending, it can be repurposed to answer novel questions without need for cleansing or interpretation steps.

Without documentation of the whole pipeline-the discussion and publication of tools, data storage, representation decisions, and the complete initial dataset $9-$ everything published in an Open Data format must be taken on trust, it is harder to understand what new observations could be added to that data. We acknowledge that archaeological projects over multiple field seasons can be incredibly complex, even assuming decisions are well documented in the heat and mud of the moment. Documentation should be sufficient to render the data commensurate, with more specific details in Section 5.3. Outside of test driven development, the only heuristic in documentation should be that of contributing to it. The main difficulty here will be adopting habits and project management practices which make this sort of exercise possible.

For mature projects, culling raw data down into well described datasets with a discussion of how the published data was chosen for inclusion is already practiced by the editors of data journals. In this regard, project management and new documentation practices must start at the dataset preparation stage. In this "bolt-on" (Marwick. 2017) mode, documenting the data cleansing process can clarify why items were removed or certain controlled vocabularies were chosen after the fact.

Unfortunately, when a project is in bolt-on mode, it can tend to be more a matter of funding, regulatory, or institutional compliance than a desire to bring a legacy dataset up to usable standards. When in this mode, these legacy datasets only offer

${ }^{9}$ See (Wilkinson et al 2016 p.18-19) for a set of resources exploring the value of this additional documentation. 
the barest minimum of description around their date 10 Without description or clean data, or any hints on how the original researchers used the data, the only use of the data is to display itself: the spreadsheet can be loaded and viewed, but not used. Not much documentation is needed, as a minimum (as we will discuss later): licensing, a description of each column's contents and if there are any controlled vocabularies is sufficient to allow some reuse of the data. More description is certainly better, but not practical in many cases.

\section{Research Programs}

Differentiating between science and pseudoscience is a question of utility and reliability of the research. Open Data, we suggest, has a similar differentiation. The demarcation problem in the philosophy of science differentiates science from pseudoscience. In this chapter, we borrow vocabulary from Lakatos' answer to this problem. To be clear, while we are applying the language of the philosophy of science to the problem of dataset quality, we are not claiming that a poor dataset makes a research program pseudoscience. Application of the many different philosophies of science to the various arguments of the practice of archaeology is outside the scope of this chapter.

Lakatos notes the challenge of defining a research program:

Knowledge in Latin is scientia, and science came to be the name of the most respectable kind of knowledge. But what distinguishes knowledge from superstition, ideology, or pseudoscience? The Catholic Church excommunicated Copernicans, the Communist Party persecuted Mendelians on the ground that their doctrines were pseudoscientific. (Lakatos 1978)

Frequently, scientific discovery can be contrary to authority or popular consensus, and relies on statements which contradict established facts. With online resources, we do not need to be content with published, established, knowledge. Instead, we can interrogate those assertions of knowledge via the data and methods which produced them. When granting agencies require open publication of data for this purpose, data is commonly published without documentation to address questions of its utility, reliability, or reusability.

Lakatos describes a good research program as progressive. A progressive research program can, "predict novel facts, facts which had been either unthought of, or have been "contradicted by previous or rival programmes," (Lakatos, 1978). In contrast, a bad program is degenerate. These degenerate research programs refer to pesudoscientific research where, "theories are fabricated only in order to accommodate known

${ }^{10}$ To explore this bolt-on world, perform a dataset search on core.tdar.org with the search term of "Excel". 
facts," (Lakatos. 1978). We can use the terms progressive and problematic (Lakatos' "degenerate") to also apply to published datasets.

A progressive dataset requires more than its publication in raw form. It must be able to answer novel questions: questions not anticipated during creation of the dataset. It must be able to be reproduced. That is, following the collection and analytical methodologies articulated in the Open Data's metadata, we must be able to collect a new dataset which reaches similar conclusions.

Datasets that do not provide clear documentation for their conclusions (i.e. problematic Open Data) will require some sympathetic magic in order to be reused. As a simplistic and extreme example, consider that of Esoteric Egypt:

... [a] vision of ancient Egypt replete with arcane knowledge and significance. This view has been elaborated at odds with scholarly Egyptology by people who believe that they can see a great deal more hidden inside the Egyptian heritage than the scholars do, by virtue of their openness to mystic, symbolist, or some other highly complicated avenue of interpretation (Jordan 2006).

Hypothetically, any datasets produced by these pseudoscientists encode their mystical assumptions into what they collect. It would be virtually impossible to simply reuse their data (if even published openly) in a progressive research program. Problematic datasets allow only for the rearrangement of what is known into expected outcomes. They provide, and allow for, a profound lack of novelty, concealing a lack of substance protected by appeals to authority. Problematic research programs follow the sympathetic law of magic: like produces like. While the demarcation of a research programme as degenerate instead of progressive is a discussion for the philosophy of science, the output of these problematic practices can, itself, only be of little utility whatsoever.

\section{Progressive and Problematic Datasets}

The use of Open Data requires more than just the data. With progressive Open Data, the reusers must be able to reconstruct how the data was made and what it means in practice to the people who recorded it 11 Through comments, metadata, documentation, and published code, we must be able to explore and reconstruct the knowledge and methods of the recorder at the time of recording. There exist multiple ways to understand and apply data, and mistaking someone's subjective recordings for

\footnotetext{
${ }^{11}$ Shamus Young's blog has a wonderful reflection on the purpose of documentation: "It's pretty common to write code and then forget what it does or how it works. So when you're writing code you should always be thinking of the other programmer. The dumb programmer. The programmer who doesn't understand this code and has no idea what's going on. The odds are very good that this other programmer is going to be a future version of you," (2013). The utility of these practices is that they will predominantly benefit the most frequent user of the data: the original producer.
} 
objective measurements can lead to painful misapplications (Ballsun-Stanton, 2012. p. 338). Documented source code and automated test-driven methods of analysis provide an unambiguous basis for reconstructing the interpreter's point of view on the appropriate use and interpretation of the recorded signs (Peirce. 1931) Publication of a progressive dataset requires that the author's original and tacit knowledge of the data's creation and methods of use are made as explicit as possible. Turning analysis into an automated output with good software development, preregistration, and quality assurance testing serves to make many tacit assumptions about the analysis an explicit part of the analysis methodology. This sort of automation also promises some efficiency gains: when an edit is desired to some portion of the output, the source code can be run again with that small fix in place, instead of needing to manually regenerate all the graphs and charts that draw from the newly changed data.

Coding and documenting research methodologies and analyses to a point where other researchers can employ them without assistance allows authors to reuse not only the data, but the tools used to manipulate that data (see Lowndes et al, 2017). This possibility for reuse eases work in future projects, increases reliability, and allows novel facts to be generated by them through the work of other researchers.

While subjectivity and objectivity in research are highly contested topics, we can note that the demarcation we are proposing is equally valid between qualitative and quantitative data. A field notebook, carefully scanned and annotated can easily qualify as a progressive dataset. Tagging of terms and capture of the methodology for recording observations within the notebook allows for reuse of the data and continued observations in the same style.

Problematic Open Data, on the other hand, presents the data as fait accompli. Any metadata which does exist serves to tautologically describe columns (e.g. a description empty of meaning and means of linking or comparing with other published datasets: "The column labelled Area Shards should always be the Shards found in the survey area." lacks a measure of unit area or any discussion or pointers to methodology.) While these tautological comments are better than nothing, they fail to address the fundamental epistemological question: "which data belongs in this column and what should not be in this column?" They also lack necessary metadata like units, definitions of a controlled vocabulary, or discussion of allowed domain. Codd defines a column's domain as: "the set of all those values which can ever occur in a given column," 1980 . For the example above, the lack of instruction for the unit of measurement or scale would make it difficult to understand the data. The domain defines everything a value of a certain type is allowed to be. Problematic data only defines the domain by what data is present, not the boundary conditions for determining allowed and erroneous data. Therefore, it is functionally impossible to append new data to the dataset (or to integrate it into new research).

One method of demarcation between progressive and problematic Open Data has two tests: 
1. Is the re-user of data in a position to add data to the dataset?

2. Can the re-user of data re-run the initial data analysis of the author without knowing the conclusion of the original work?

A progressive dataset is capable of re-running an analysis by following the methodology without knowing what the outputs should be. This independent interrogation allows the data to be open to robust questioning. To the contrary, a problematic dataset is usable only when the claims of its creators are fully known. While it can be used to support those claims, extending it beyond the problem at hand is virtually impossible. With problematic datasets, unless the user knows ahead of time what is should be proven, it becomes difficult to reproduce the results. Here in the worst cases of like-produces-like, researchers write the bottom line first, and then figure out ways to justify it.

\subsection{An Example of Problematic Open Data}

Let us consider an example from tDAR by McGovern (2003) 12. This dataset is chosen as a representative example of uploaded data that is problematic from our perspective. While it is a component of a larger project, the North Atlantic Biocultural Organization, it is published as its own dataset and we will assess it in that light.

We start our assessment by looking at the metadata. The dataset has a title of: "AST rework," with a summary "Excel workbook with spreadsheets and charts by TMG," McGovern (2003). We can assess the metadata against the specific FAIR principles (Findability, Accessibility, Interoperability, and Reusability) using heuristics from Hagstrom (2014). The resource has a DOI, and is theoretically findable. However, the summary metadata does not allow us to differentiate this result's contents from any other Excel Workbook, so it would be difficult for a researcher entering domain specific search terms to arrive at this item. By virtue of being published on tDAR, the resource is accessible. tDAR implements an archive that has a high level of reliability (Center for Digital Antiquity, 2015). The metadata are not interoperable because no metadata besides the summary and attribution information are published. And the metadata alone prohibit reuse because there is no clear license.

Inspecting "Sheet 3" (Figure 1), we have what is likely a count in two columns. In the second pass of analysis, when we are inspecting the data, it is problematic because the columns violate Codd's idea of domain: there are no column headers, and the

12 https://core.tdar.org/dataset/394057/ast-rework Archive: https://perma.cc/ WA4N-24R9 We would like to apologise to Thomas for using such an old example of his work as our worked example. He has uploaded many items to tDAR and our example is absolutely cherry picking an old example for demonstration purposes only. Our discussion here should not be taken as a criticism of his work or research, but using an example of an ancient uploaded file for the education of all. 
Fig. 1 Sheet 3 from McGovern (2003). This sheet from an

Excel workbook demonstrates many issues of a problematic dataset in a space small enough for a screenshot and is not representative of the quality of the full publication. Original is available as an

Excel worksheet at https:

//core.tdar.org/dataset/ 394057/ast-rework

\begin{tabular}{l|r|}
\hline \multicolumn{2}{l|}{ AST Settlement Period } \\
\hline Cattle & 1 \\
\hline Horse & 3 \\
\hline Pig & 118 \\
\hline Sheep/Goats & 32 \\
\hline total domesticate & 154 \\
\hline & \\
\hline Walrus & 7 \\
\hline Fish Sp. & 2 \\
\hline Mollusca & 6 \\
\hline \multicolumn{1}{|c|}{ total NISP } & 169 \\
\hline & \\
\hline LTM & 3 \\
\hline MTM & 16 \\
\hline UNI & 4918 \\
\hline & total TNF \\
\hline
\end{tabular}

content of column one is either: a semantically significant null (intentionally blank line) differentiating the data tables, the name of the unit being counted, an acronym that isn't part of a controlled vocabulary in the metadata, or a label for a calculation. In column two, we have three different sorts of values: counts, summation, and percentage. While this chart is quite useful within the context of the reports it was generated for, the data under analysis cannot be reused in other research as we have no sense of how the data was collected or what units of measurement were used for sampling. Furthermore, we cannot even load this data into another program to use as part of a different piece of research because of the domain problems noted earlier. Another troubling indication of a problematic dataset is the use of visual formatting to indicate domain change. The totals have thick bars over them and right-align formatting on column one. The calculation is set off by a thick border around all sides. To reuse this data, either requires human intervention or the parsing program must be able to programmatically identify formatting and assign meaning to that formatting, a tedious task indeed.

The Excel worksheet does have the advantage of showing the analysis work on the data, which can help future users of the data explore the author's intended use of the data. Despite this advantage, we would have to describe this dataset as problematic. This data cannot be reused in other research on North Atlantic biology and cannot be extended, because looking at this dataset alone, there is no way to collect commensurate samples with similar duration, region, or methodology. 


\subsection{Usable Open Data}

In order for data to be used, data must be usable. For example, usable data can:

- be easily transformed into other formats;

- be easily sorted and filtered;

- be easily referenced into extant lookup domains (places, people); and

- be easily aggregated into summary statistics.

A good element (though certainly not the only indicator) of a usable dataset is one that employs Linked Open Data (LOD). LOD references stable external Uniform Resource Identifiers (URIs) as consistent keys for the data. This consistency provides a historical and communally agreed upon pattern for ways of representing the observed/recorded data. While there are many other benefits of LOD (Bizer et al, 2009), as a basic indicator of data quality, the use of LOD indicates that some thought has been given to the reuse of data. As with any long-term website, the dynamics of a singular LOD identifier can change over time: dying, becoming stale, being updated with a different version, or other problems are all aspects of the pragmatic LOD infrastructure on the web as it exists (Käfer et al, 2013). However, the singular advantage of the LOD URI is that it serves as a unique and shared identifier which does not depend on the original site for its meaning. While the site hosting the URI may die, the fact that the identifier is used in the same way by all documents allows for the data from these independent sources to be linked and rendered commensurate. While the hosting site adds richness and nuance to the identifiers, it does not serve as a single point of failure. Therefore, there is a notable advantage of using LOD identifiers even taking into account the inevitable change and death of some of their hosting sites. URIs should be stable, but with the use of version control, systems which use URIs can update their concepts with a clean and completely reversible mapping from new to old schema. Datasets can be modified to remain useful, but keep their historical versions available for inspection.

While the preparation of a LOD export is an indicator of quality, a more thorough indicator of a progressive dataset would be providing the Extract, Transform, and Load (ETL) process (Jacobs, 2009) used to prepare the research data for publication. Publication of the ETL process entails both the programming code used to convert data and documentation on the code's use (see Kansa and Kansa, 2010, Kansa et al, 2014). The documentation and test suites of the code indicate why these choices were made and the reasons for reuse. Problematic datasets here are those which cannot be linked, manipulated, sorted, or filtered. Progressive datasets can be normalized and reinterpreted without risk of corruption. 
Fig. 2 Screenshot of a record from Russell et al (2013). Note that each variable name is a link pointing to a description of the variable. The controlled vocabulary values are also linked, going to descriptions of all allowed vocabularies in the column

\begin{tabular}{l|l} 
Main Observation & Standards Annotations \\
I Descriptions (19) & \\
\hline Descriptive Variable & Value(s) \\
\hline Element & Scapula \\
\hline Taxon & Capra \\
\hline Symmetry & Left \\
\hline Letter Code & F \\
\hline Find Number & 1 \\
\hline Validity & Measurements \\
\hline Recovery & 4 mm dry sieve \\
\hline Certainty & Probable \\
\hline Weathering & Slight weathering
\end{tabular}

We can briefly examine a record from the zooachaeological dataset by Russell et al, the product of the above published process. While this output is orders of magnitude more ornate (and expensive) than McGovern's Excel sheet, they are both tables of counts relating to animals. If we examine a specific record within the table (See Figure $2 \|^{3}$, every variable (what would be a column header in the table) is a link. These links go to a controlled vocabulary describing the variable. More importantly, other datasets use these URIs, and so data from this table is commensurate with data from other tables. The methodology of this project is discussed both within the same website, and in Kansa et al (2014). Using their documentation, it is theoretically possible that we could meaningfully add data to this dataset without reducing its validity.

\subsection{The value of documentation and test-driven development}

To reduce errors, increase confidence in outputs, and increase the reusability and reproducibility of the code which analyses their data, researchers should engage in test driven development (Beck, 2003) of their data analysis suites. Test driven development implores programmers to write tests for their software before they write their software. One source of frustration is the delay between performing the analysis and writing about the analysis performed. Researchers should create tests for their analysis workflow before they perform the analysis, regardless of the mechanism employed. These tests, documented with their justifications, citations, and expected outputs, form a coherent and peer-reviewable report of analytical methods before the analysis occurs. With this documentation, project write-ups (some years later) will not be an exercise in uncomfortable reconstruction of decisions.

13 https://opencontext.org/subjects/E93B0801-C1F4-441F-8A87-3E55381CF23E Archive: https://perma.cc/Z6JW-7NM5 
Test driven development implies the development of code to perform analyses. While there are opportunities available to researchers wanting to learn how to code $1{ }^{14}$ research software development is not for everyone. Developing this workflow presents an excellent opportunity for cross-faculty collaboration with statistics or computing departments, or an excellent job for a Master's student (see Spring et al, 2018). By insisting on test driven development, the lead researcher of the project can gain confidence that the code commissioned from other people is performing the correct analysis. This quality assurance process is a means by which all researchers, not just coders, do not need to take the quality of research software's outputs on faith. Moreover, the same testing methods can be applied (with more difficulty) to excel spreadsheets, providing an opportunity to detect potentially hugely embarrassing bugs before articles go to print (see Giles, 2014: Worstall, 2013).

An intentional quality assurance process also allows for performing quality assurance on datasets (Kansa and Kansa. 2010. Section 3.1). Creating a series of software tests which can raise alarms when incoming data is outside of parameters provides a mechanism for making sure any typos or methodological misunderstandings can be caught in the field. Creating born-digital archaeological data is its own extensive undertaking (see Ballsun-Stanton et al, 2018), but the idea of an explicit quality assurance checklist, preferably software based, can be applied to any data collection methodology. All it needs is a checklist of a series of very easily confirmed or falsified things and the intentional application of that checklist to all or a random subset of incoming data. The practice of applying the checklist instead of simply an expert eye on area supervisor's daily notes means that small details that might be missed due to exhaustion are now routinely checked because of their presence in the checklist (Hales and Pronovost, 2006).

Test driven development also encourages explanatory comments in the code itself. This commentary provides documentation of assumptions made in the code and the expected failure modes of unclean data or bugs in the analysis. This quality assurance methodology also articulates the data's domain. It indicates what must qualities data or code must have to pass or fail for the data collection or analytical programming to be considered reliable. As well, we gain the confidence of not needing to trust the authors' description of their intention, as the code provides explicit annotation of their workflow, and what outcomes were considered correct or problematic.

Problematic datasets are usually published without any internal quality assurance testing or documentation of how they were created. We must take their authenticity on trust and it is quite likely that their research methods conceal flaws. For example, a program called statcheck (Epskamp and Nuijten, 2016) which: “... extracts and checks statistics from psychology research papers-and it has confirmed that the literature

${ }^{14}$ Ballsun-Stanton is an instructor for Software Carpentry (https://sof tware-carpentry.org/ Archive: https://perma.cc/3ALD-PBMV) and encourages all researchers wanting to find out more about computational analysis to find local workshops in their area. Software carpentry is an excellent and gentle introduction to software based data analysis (Wilson. 2014). 
contains plenty of errors," (Baker. 2015), regularly scans published literature and posts embarrassing reviews. By increasing focus on testing their analyses before use, authors can avoid being caught out by the most trivial automated checking. The authors are aware of no significant meta-analyses looking at error rates in published archaeological data, but considering the stress and difficulty of fieldwork such studies might have a significant chance of finding typos and obviously problematic data. Because archaeology tends to destructive research, it is difficult to find errors in data at any significant remove from the field. Software tests and checklists present a means of applying other disciplines' quality assurance processes to fieldwork: thereby reducing sources of frustration during publication.

A progressive dataset is well documented and reusable without consultation. Adding to a dataset or combining datasets for the generation of novel facts is much easier than the ab novo creation of a dataset and the mechanism of its creation and analysis. Research is incredibly expensive and significant funds are spent on the development and deployment of use-once infrastructure: write-once read-once spreadsheets, GIS sets, tables in Word, etc. While the data from the world is unique, the means of analysis should not be. Again, to emphasise, we are not recommending qualitative or quantitative approaches here. While longhand journals suggest different kinds of analysis, their results must still tell a persuasive story. The documentation of method and data quality testing along with a plan of analysis and publication can create data that other researchers with similar methods can inspect and reuse. Legacy data presents a problem for some aspects of archaeology, as there is no way to go to the field as it was $x$ years ago. However, in the process of preparing a legacy dataset, it is possible to develop data quality tests for obvious typos and conversion schemes for vocabulary items onto LOD URIs even after the fact (Kansa and Kansa, 2010. s. 2.1). Extracting, transforming, and loading legacy data can itself be an area of research, or the topic of a class or degree.

If we can reuse data as well as analysis mechanisms, we can achieve greater and more reliable research impacts for the same initial set of money, and less overall time and labour than a typical legacy data project. If we publish data without plans for its reuse, the publishing serves little purpose. Using test driven development to create our analysis platform creates more reliable outputs, with less time spent on iterating quality via peer review and less time spent on reinventing the wheel. Using public methodological repositories allows for greater and earlier public feedback by reducing errors and avoiding retractions. Using other peoples' data with our own innovations can generate novel ideas without needing to spend months writing grants. With a community working on open science, we get more papers and more confidence in our results for the same money. 


\subsection{Avoiding implicit knowledge transfer}

Academic knowledge and expertise exists not only in papers, but in the lived experiences of people (Galison 1997). Currently, most data is presented with the implicit knowledge of the intended use of the data. As a discussion on data sharing in Nature notes:

Researchers generally create their own formats because they believe that they know how their users want to use the data," says [James] Boyle. But there are roughly a billion people with Internet access, he says "and at least one of them has a smarter idea about what to do with your content than you do (Nelson 2009).

We must not confuse the novelty of the research data with the novelty of the research methods or even the analysis. When we, as researchers, produce open data, our expectation is that this data may be reused by our colleagues. Therefore, if we take Boyle's point, it is necessary to consider the approach of Open Processing (Beck and Neylon. 2012, p. 483-484), and avoid implicit contextual expertise. Open Processing, as it is coined by Beck and Neylon, refers to the sharing of workflows and experiences alongside data itself. In essence, these workflows create our research outputs. Our data, as product of our research, is a materialized artifact of that research. It is contextualized and made useful by our methods and by our own understanding of the elements of the data (Ballsun-Stanton, 2012, p. 67). Therefore, without knowledge of the data's intended use, it becomes exponentially more difficult to untangle the data itself from its research question, and the data's capacity for reuse is significantly diminished.

Galison comments that only through the exchange of personnel (i.e. expertise) could early laboratories achieve replication of their experimental results. When trying to replicate cloud chamber imagery, “... it was only when a member of the Bristol team of emulsion experts arrived in Berkeley that the Berkeley team succeeded in making its emulsions exhibit the tracks of pions," 1997. While we no longer require the movement of personnel and material between research labs to spread these new techniques, much of research progress still benefits from the exchange. In these electronic times, we have more easily portable tools: remote code repositories, virtualised runnable images for the entire process, video, and technical documentation with metadata. These tools allow people to extend, replicate, use methods from peer-reviewed sources, and to extend their own mechanisms of experimentation. Test driven development, as well as documentation of analysis and dataset preparation, allows for the creation of novel facts by other researchers without the transfer of human expertise. This independent creation is a hallmark of a progressive dataset. Problematic datasets become artifacts themselves, languishing on USB sticks and scribbles in notebooks, which require time-consuming manual interpretation to find, decipher, and use. 
As part of the Dissemination Information Packages for Information Reuse (DIPIR) ${ }^{15}$ project, researchers interviewed archaeologists and zoologists on their data sharing habits and practices. The interviews revealed an ad hoc approach to data management among archaeologists in particular, with an interviewee responding:

I use an Excel spreadsheet...which I...inherited from my research advisers...my dissertation advisor was still recording data for each specimen on paper when I was in graduate school so that's what I started...then quickly, I was like, 'This is ridiculous'...I just started using an Excel spreadsheet that has sort of slowly gotten bigger and bigger over time with more variables or columns...I've added...colour-coding...I also use.... a very sort of primitive numerical coding system, again, that I inherited from my research advisers...So, this little book that goes with me of codes which is sort of odd, but...we all know that a 14 is a sheep, Kansa and Kansa 2013).

14 as a sheep is the sort of tacit, non-extendable, and non-reproducible symbol that marks a dataset as problematic for Open Data. The self-referential and opaque vocabulary shorthand in this collection has no tested analyses being performed before the data is collected. Instead, the coding is informed by the conclusions which they seek to test. This process is scientific, as they seek to statistically support their hypothesis, but is not good data practice.

The data was not created with the foresight of open sharing, thus the abbreviations in the data only support the specific research project at hand. It would be unimaginable — or at least beyond the scope of many budgets - to add to this dataset, or to clean it to such a degree that its data could be used in a different project. And, if this dataset was to be cleaned up and published, it would only be through the efforts of those who had worked to collect it in the first place. They would have to use their memories of collecting the data to add sufficient contextualization for other people to use it. Framed within the research question of the original investigators, this kind of self-reflexive emendation is sufficient to produce statistical aggregate outputs suitable for a book or other publication, but not sufficient for other research projects to do the same.

\subsection{The Nature of Progressive Open Data}

Progressive Open Data is FAIR (Wilkinson et al, 2016). When looking at it as a set of documents, that set is Findable, Accessable (to authorised users through a system which does not rely on someone checking their email), Interoperable (with csvs or other machine readable formats normalised and tested against some external standard), and Reusable such that other researchers can ask questions of the data that the original researchers did not necessarily intend.

15 http://www.oclc.org/research/themes/user-studies/dipir.html Archive: https:// perma.cc/5822-396R 
Progressive Open Data collections support Generativity, Legibility, and Creativity (Padilla, 2017). These datasets allow other researchers to generalise and generate conclusions, meet data quality standards (and have automated checks of those standards as per test driven development above), and feel safe in using the data for their own creative ends.

To will reiterate some design patterns to make these Progressive datasets:

- Build data collection methods and an analysis pipeline before going out to the field. Try to preregister what aspects of the methodology or analysis are sufficiently generalised to support testable research instead of post hoc conclusions (Nosek et al, 2018, 16.

- Submit data as a data paper for peer review. Open Context and the Journal of Open Archaeological Data support data papers, short 2000-3000 word papers which describe and defend a published dataset

- Find a Master's student who wants a thesis or $\mathrm{PhD}$ in want of a paper and get them to reproduce the study, given the hypotheses, intended analyses, and data, just as an external researcher would be able to obtain from reading papers and downloading datasets. The points where the student comes to the original researchers for assistance should be noted to see if they represent flaws in the data description or teachable moments (Spring et al, 2018).

- Try to use other peoples' data in support of the current project's hypotheses or areas of exploration. This exploration is an excellent source of conference presentations or journal articles.

- Try to export project data as JSON or XML and then convert back to CSV. While data can be converted without trouble, spreadsheets created for human consumption will not fare well in translation.

- Use Linked Open Data identifiers where possible. When preparing data for export, write scripts to convert from internal identifiers to Linked Open Data. The various Getty Vocbaularies 17, Research Vocabularies Australia 18, and Schema.org19 ontologies all hold well formed vocabularies which might be able to fit some

\footnotetext{
${ }^{16}$ For more on preregistration, expect a chapter soon about "Analysis before the Spade" by Shawn Ross and Brian Ballsun-Stanton at http://dhainpractice. anthropology .msu.edu/ 17 http://www.getty.edu/research/tools/vocabularies/ Archive:https://perma.cc/JF6G$\mathrm{X} 64 \mathrm{~V}$

18 https://www.ands.org.au/online-services/research-vocabularies-australia Archive: https://perma.cc/CA92-346F

19 Archive: https://perma.cc/D887-KXYD
} 
of research project's controlled vocabularies 20 For qualitative journal research, annotations and tagging of journal entries after the fact can make each written entry findable by others and far richer in its ability to draw in and reference other research.

In general, all of these recommendations resolve to the following advice: ask other people to use your data to support their own research. Regardless of its quantitative or qualitative nature, well formed data can be put to many uses by many people. Good datasets can also be a source of academic credit, cited in the same fashion as any other published work.

\section{Conclusion}

[A]nd the gross failures of past prophets to predict what lay immediately ahead of them, could be described as 'Failures of Nerve'...Any sufficiently advanced technology is indistinguishable from magic, Clarke 1974 p.30,39).

Treating Open Data as sympathetic magic, as Clarke points out, is a "Failure of Nerve." The researcher, with the facts and capabilities arrayed before them shrinks back and presents the inoperable image instead, hoping that it will stand in for the thing itself. They treat their data like Clarke's failed prophets: not able to imagine that others would be able to utilize it to answer novel questions.

Open Data provides a great foothold from which we can grow scientific research and ensure that communities of researchers have access to irreplaceable research data. Currently, there is an emphasis on the act of sharing in regards to Open Data. This conversation should remain at the forefront as we move forward in our data sharing practices. However, as researchers, we are set to benefit from a more open Open Data. As we continue these discussions, what we as a community would benefit from is bringing forward a discussion of the nature of things we share. Marwick (2017) is an example of computational data reuse in archaeology, using other datasets to test a hypothesis. Strupler and Wilkinson (2017) is an example of the application of some techniques from Open Science to fieldwork. Many of us are working towards clean, interopoerable, archaeological data. Hopefully some of the techniques we have presented in this chapter can help differentiate useful data management practices from problematic ones (see Arbuckle et al, 2014; Marwick, 2017; Lowndes et al, 2017; Sobotkova, 2018).

Open Data can, and should, include documentation of analysis and workflows as to open new avenues of research for the community and better support reproducibility

${ }^{20}$ As a note, if a vocabulary's set of terms match most, but not all of the research project's controlled vocabularies, this is an opportunity for a paper and a contribution to that vocabulary, not a reason to reject the use of the controlled vocabulary. 
in archaeology. Currently, most Open Data systems support some metadata requirements. Metadata helps other researchers understand datasets but does not support communication of workflow or analysis. Without documentation that outlines workflow or analysis, researchers limit their data potential. By documenting only metadata, it can also hide tacit assumptions about the data that we may not even be aware of. As a result, researchers can find and look at this data, even reuse the data, but not replication of workflow or analysis. Even data reuse is made a more challenging task, as there is no documentation of assumptions or tacit knowledge. Testing the quality of documentation is an excellent exercise for welcoming new graduate students without risking a hostile paper critiquing a published dataset. Nevertheless, if one researcher makes incorrect assumptions of the workflow or analysis of another's data, they risk misuse or misunderstandings. With a lack of quality control, we cannot be sure we are not engaging in a kind of sympathetic magic as like produces like.

Sharing the means of knowledge production is an incredibly valuable avenue for us as researchers to pursue. Organizing data to a standard where it is easy to reuse is no small task; documenting the way we produce and analyze our data adds even more work. But, by making available documentation of workflow and analysis, other researchers can not only reuse data but also reuse and replicate methods of working and analysis. By doing so, archaeologists can engage in more rigorous science. As a science that engages in unreproducible experiments, sharing our workflows and analysis better equips our efforts towards reproducibility. It also opens our data to faster integration into other datasets, making our data more valuable and more equipped to answer research questions of broader scope.

\section{References}

Arbuckle BS, Kansa SW, Kansa E, Orton D, Çakırlar C, Gourichon L, Atici L, Galik A, Marciniak A, Mulville J, Buitenhuis H, Carruthers D, De Cupere B, Demirergi A, Frame S, Helmer D, Martin L, Peters J, Pöllath N, Pawłowska K, Russell N, Twiss K, Würtenberger D (2014) Data sharing reveals complexity in the westward spread of domestic animals across neolithic turkey. PLoS One 9(6):e99845

ArcheoInf-Redaktion (2010) ArcheoInf. URL https://perma.cc/3SZ6-AAQM, accessed: 2018-11-23

Australian Research Council (2016) Research data management. https://perma. Cc/F2UL-7YM8, accessed: 2016-10-14

Baker M (2015) Smart software spots statistical errors in psychology papers. Nature News

Ballsun-Stanton B (2012) Asking about data: Exploring different realities of data via the social data flow network methodology. PhD thesis, University of New South Wales

Ballsun-Stanton B, Ross SA, Sobotkova A, Crook P (2018) FAIMS mobile: Flexible, open-source software for field research. SoftwareX 7:47-52, DOI 10.1016/j.softx. 
2017.12.006

Beck A, Neylon C (2012) A vision for open archaeology. World Archaeol 44(4):479_ 497

Beck K (2003) Test-driven Development: By Example. Kent Beck signature book, Addison-Wesley, Boston, MA

Bizer C, Heath T, Berners-Lee T (2009) Linked data: The story so far. In: Sheth A (ed) Semantic Services, Interoperability and Web Applications: Emerging Concepts: Emerging Concepts, Information Science Reference, pp 205-227

BMC Psychology (2017) Results-free review: a new model of peer review. URL https://bmcpsychology.biomedcentral.com/about/ results-free-peer-review-for-authors, accessed: 2018-11-28

Carver G, Lang M (2012) Reflections on the rocky road to E-Archaeology. In: Graeme Earl, Tim Sly, Angeliki Chrysanthi, Patricia Murrieta-Flores, Constantinos Papadopoulos, Iza Romanowska and David Wheatley (ed) Archaeology in the Digital Era, dare.uva.nl, pp 224-236

Center for Digital Antiquity (2015) How to ensure proper curation of digital archaeological records. Tech. rep., Center for Digital Antiquity

Clarke AC (1974) Profiles Of The Future. Victor Gollancz Ltd, London

Codd EF (1980) Data models in database management. In: Zilles SN, Brodie ML (eds) Proceedings of the 1980 workshop on Data abstraction, databases and conceptual modeling, ACM, New York, NY, USA, vol 16, pp 112-114

Costa S, Beck A, Bevan AH, Ogden J (2013) Defining and advocating open data in archaeology. In: Archaeology in the Digital Era, Amsterdam University Press, Amsterdam, Netherlands, pp 449-456

Edmunds S (2016) Reproducible research resources for research(ing) parasites. URL https://perma.cc/EHY2-4GNK accessed: 2016-8-15

Epskamp S, Nuijten MB (2016) statcheck

Findley MG, Jensen NM, Malesky EJ, Pepinsky TB (2016) Can Results-Free review reduce publication bias? the results and implications of a pilot study. Comp Polit Stud

Flannery KV (1982) The golden marshalltown: A parable for the archeology of the 1980s. Am Anthropol 84(2):265-278

Frank RD, Yakel E, Faniel IM (2015) Destruction/reconstruction: preservation of archaeological and zoological research data. Arch Sci 15(2):141-167

Frazer JG (1922) Golden Bough: A Study in Magic and Religion, One Volume, Abridged Edition. Macmillan Company, New York, USA

Galison P (1997) Image and Logic: A Material Culture of Microphysics. University of Chicago Press, London, UK

Giles C (2014) Data problems with capital in the 21st century. Financial times 23, URL http://blogs.ft.com/money-supply/2014/05/23/ data-problems-with-capit

Hagstrom S (2014) The FAIR data principles. URL https://www.force11.org/ group/fairgroup/fairprinciples, accessed: 2018-11-30

Hales BM, Pronovost PJ (2006) The checklist-a tool for error management and performance improvement. Journal of critical care 21(3):231-235, DOI 10. 
1016/j.jcrc.2006.06.002, URL http://dx.doi.org/10.1016/j.jcrc.2006. 06.002

Horbach SPJMS, Halffman WW (2018) The changing forms and expectations of peer review. Research integrity and peer review 3:8, DOI 10.1186/s41073-018-0051-5, URL http://dx.doi .org/10.1186/s41073-018-0051-5

Jacobs A (2009) The pathologies of big data. Commun ACM 52(8):36-44

James L (2013) Defining open data. https://perma.cc/4RMX-ET3Y, URL https://perma.cc/4RMX-ET3Y accessed: 2018-11-23

Jordan P (2006) Esoteric egypt. In: Fagan GG (ed) Archaeological Fantasies: How Pseudoarchaeology Misrepresents the Past and Misleads the Public, Psychology Press, pp 109-128

Käfer T, Abdelrahman A, Umbrich J, O'Byrne P, Hogan A (2013) Observing linked data dynamics. In: The Semantic Web: Semantics and Big Data, Springer Berlin Heidelberg, pp 213-227, DOI 10.1007/978-3-642-38288-81_15, URL http:// dx.doi.org/10.1007/978-3-642-38288-8_15

Kansa E (2012) Openness and archaeology's information ecosystem. World Archaeology 44(4):498-520

Kansa E, Kansa SW, Arbuckle B (2014) Publishing and pushing: Mixing models for communicating research data in archaeology. IJDC URL http://www . ijdc . net/index.php/ijdc/article/view/301

Kansa EC, Kansa SW (2010) Publishing data in open context: Methods and perspectives. Center for the Study of Architecture Newsletter 23(2), URL http: //csanet.org/newsletter/fall10/nlf1001.html

Kansa EC, Kansa SW (2013) We all know that a 14 is a sheep: Data publication and professionalism in archaeological communication. Journal of Eastern Mediterranean Archaeology and Heritage Studies 1(1):88-97

Kersel MM (2016) Response: Living a semi-digital kinda life. In: Averett EW, Gordon JM, Counts DB (eds) Mobilizing the Past for a Digital Future: The potential of digital archaeology, The Digital Press, University of North Dakota, Grand Forks, pp 475-492

Lakatos I (1978) Science and pseudoscience. BBC Radio Talk, URL http://www.lse.ac.uk/philosophy/department-history/ science-and-pseudoscience-overview-and-transcript/

Lowndes JSS, Best BD, Scarborough C, Afflerbach JC, Frazier MR, O'Hara CC, Jiang N, Halpern BS (2017) Our path to better science in less time using open data science tools. Nature ecology \& evolution 1(6):160, DOI $10.1038 / \mathrm{s} 41559-017-0160$

Lynch C (2008) Big data: How do your data grow? Nature 455(7209):28-29

Marwick B (2017) Computational reproducibility in archaeological research: Basic principles and a case study of their implementation. Journal of Archaeological Method and Theory 24(2):424-450, DOI 10.1007/s10816-015-9272-9, URL http://dx.doi.org/10.1007/s10816-015-9272-9

McGovern T (2003) AST rework URL https://core.tdar.org/filestore/ get/394057/360651

Nature (2008) Community cleverness required. Nature 455(7209):1 
Nature (2016) Reality check on reproducibility. Nature 533(7604):437, DOI 10. $1038 / 533437 \mathrm{a}$

Nature Publishing Group (2018) Availability of data, materials, code and protocols. https://www.nature.com/authors/policies/availability.html. URL https://www.nature.com/authors/policies/availability. html, accessed: 2018-11-28

Nelson B (2009) Data sharing: Empty archives. Nature 461(7261):160-163

North M (2006) Protecting the past for the public good: Archaeology and australian heritage law. PhD thesis, University of Sydney

Nosek BA, Ebersole CR, DeHaven AC, Mellor DT (2018) The preregistration revolution. Proceedings of the National Academy of Sciences of the United States of America 115(11):2600-2606

Open Knowledge International (2016) Open definition 2.1. http:// opendefinition.org/od/2.1/en/, URL http://perma.cc/NJ2K-QFT4. accessed: 2017-10-18

Open Source Collaboration (2015) Estimating the reproducibility of psychological science. Science 349(6251)

Padilla T (2017) On a collections as data imperative. UC Santa Barbara URL https : //escholarship.org/uc/item/9881c8sv

Peebles GM (2010) Sharing data, swapping knowledge, and building community: Moving archaeology from deep storage into the public eye through the internet. In: Frischer B, Crawford JW, Koller D (eds) Proceedings of the 37th International Computer Applications and Quantitative Methods in Archaeology Conference, Archaeopress, Oxford, BAR International Series, pp 298-307

Peirce CS (1931) Collected Papers. Harvard University Press, Cambridge, MA protocolsio (2018) protocols.io - the \#1 science methods repository.https://www . protocols.io/, URL https://www.protocols.io/ accessed: 2018-11-23

Russell N, Twiss K, Frame S, Zooarchaeology Ç, Yeomans L, Martin L, Christensen C, Orton D, Demirergi A, Meese S, Pawlowska K, Aydinuloglu B, Henton L, Watson A, Mayon-White R, Cameron I, Erwin A, Dimitrijevic V, Daly R, Symmons N, Yeni S (2013) Çatalhöyük main zooarchaeological dataset. URL https : //opencontext.org/tables/4c98ccdee589f0e76c5fa7376ac2638e, title of the publication associated with this dataset: Çatalhöyük Zooarchaeology

Sobotkova A (2018) Research compendium for a report on elenovo 2017 mound verification campaign. URL https://github.com/adivea/Elenovo2017

Sobotkova A, Janouchova P (2018) TRAP burial mound monitoring 2017. URL https://github.com/FAIMS/burial

Society for American Archaeology (2018) Editorial policy, information for authors, and style guide for american antiquity, latin american antiquity, and, advances in archaeological practice. URL http://www.saa.org/Portals/0/ SAA $\% 20$ Style $\% 20$ Guide_Updated\%20July\%202018.pdf accessed: 2018-1128

Spring J, Maller M, Mariconti E, Onaolapo J (2018) An untapped resource to reproduce studies. Archive: https://perma.cc/ 82ET-YPKH URL https://www.benthamsgaze.org/2018/07/31/ 
an-untapped-resource-to-reproduce-studies/, accessed: 2018-1127

Strupler N, Wilkinson TC (2017) Reproducibility in the field: Transparency, version control and collaboration on the project panormos survey. Open Archaeology 3(1):51, DOI 10.1515/opar-2017-0019, URL http://www.degruyter.com/ view/j/opar.2017.3.issue-1/opar-2017-0019/opar-2017-0019.xml

Vines TH, Albert AYK, Andrew RL, Débarre F, Bock DG, Franklin MT, Gilbert KJ, Moore JS, Renaut S, Rennison DJ (2014) The availability of research data declines rapidly with article age. Curr Biol 24(1):94-97

Wilkinson MD, Dumontier M, Aalbersberg IJ, Appleton G, Axton M, Baak A, Blomberg N, Boiten JW, da Silva Santos LB, Bourne PE, Bouwman J, Brookes AJ, Clark T, Crosas M, Dillo I, Dumon O, Edmunds S, Evelo CT, Finkers R, Gonzalez-Beltran A, Gray AJG, Groth P, Goble C, Grethe JS, Heringa J, Hoen PACâ, Hooft R, Kuhn T, Kok R, Kok J, Lusher SJ, Martone ME, Mons A, Packer AL, Persson B, Rocca-Serra P, Roos M, van Schaik R, Sansone SA, Schultes E, Sengstag T, Slater T, Strawn G, Swertz MA, Thompson M, van der Lei J, van Mulligen E, Velterop J, Waagmeester A, Wittenburg P, Wolstencroft K, Zhao J, Mons B (2016) The FAIR guiding principles for scientific data management and stewardship. Scientific Data 3:160018, DOI 10.1038/sdata.2016.18

Wilson G (2014) Software carpentry: lessons learned. F1000Research 3:62, DOI 10.12688/f1000research.3-62.v2, URL http://dx.doi.org/10.12688/ f1000research.3-62.v2

Worstall T (2013) Microsoft's excel might be the most dangerous software on the planet. Forbes Magazine

Yang Z, Kankanhalli A (2013) Innovation in government services: The case of open data. In: Grand Successes and Failures in IT. Public and Private Sectors, Springer, Berlin, Heidelberg, IFIP Advances in Information and Communication Technology, pp 644-651, DOI 10.1007/978-3-642-38862-0\_47

Young S (2013) Coding style part 1. URL https://perma.cc/3YGW-J6G2, accessed: 2016-10-11 